\title{
A REMARK ON COMPLEMENTED SUBSPACES OF UNITARY MATRIX SPACES
}

\author{
JONATHAN ARAZY
}

\begin{abstract}
Theorm A. Let $P$ be a bounded projection in a unitary matrix space $C_{E}$. Then either $P C_{E}$ or $(I-P) C_{E}$ contains a subspace which is isomorphic to $C_{E}$ and complemented in $C_{E}$.
\end{abstract}

1. Introduction. Let $E$ be a symmetric sequence space, i.e. a Banach space of sequences so that the standard unit vectors $\left\{e_{n}\right\}_{n=1}^{\infty}$ (defined by $e_{n}(i)=\delta_{n, i}$ ) form a 1-symmetric basis of $E$. We denote by $C_{E}$ the Banach space of all compact operators $x$ on $l_{2}$ so that the sequence $s(x)=\left(s_{n}(x)\right)_{n=1}^{\infty}$ of $s$-numbers of $x$ (i.e. the eigenvalues of $\left.\left(x^{*} x\right)^{\frac{1}{2}}\right)$ belongs to $E$, normed by $\|x\|_{C_{E}}=\|s(x)\|_{E}$. The spaces $C_{E}$ are called unitary matrix spaces. For their study see [3] and [5].

The main result of the present paper is Theorem A, stated in the abstract. It may be used in proving that certain unitary matrix spaces (the spaces $C_{p}, 1<p<\infty$, for example) are primary. The problem of whether every unitary matrix space is primary is however still open. Theorem A also has a local version which is discussed at the end of the paper.

We use standard terminology from Banach space theory, see [6]. Also we identify operators $x$ on $l_{2}$ with their matrices $(x(i, j))$ with respect to some fixed orthonormal basis in $l_{2}$. The standard unit matrices $\left\{e_{n, k}\right\}_{n, k=1}^{\infty}$ are defined by $e_{n, k}(i, j)=\delta_{n, i}$ - $\delta_{k, j}$. If $\left\{i_{k}\right\}_{k=1}^{\infty}$ and $\left\{j_{k}\right\}_{k=1}^{\infty}$ are increasing sequences of positive integers, then $Q\left(\left\{i_{k}\right\},\left\{j_{k}\right\}\right)$ is the projection defined by

$$
\left(Q\left(\left\{i_{k}\right\},\left\{j_{k}\right\}\right) x\right)(i, j)=\left\{\begin{array}{l}
x(i, j) \quad \text { if } i=i_{k} \\
0 \text { otherwise. }
\end{array} \text { and } j=j_{l} \text { for some } k \text { and } l,\right.
$$

Clearly, this projection has norm one on every unitary matrix space.

Another important projection is the triangular projection $T$ defined by

$$
(T x)(i, j)=\left\{\begin{array}{l}
x(i, j) \text { if } i<j, \\
0 \text { otherwise. }
\end{array}\right.
$$

For every symmetric sequence space $E$ let us denote

$$
T_{E}=\left\{x \in C_{E} ; x(i, j)=0 \text { if } i>j\right\} .
$$

Received by the editors August 17, 1979.

AMS (MOS) subject classifications (1970). Primary 47D15; Secondary 46B99, 47B10.

Key words and phrases. Unitary matrix spaces, symmetric sequence spaces, compact operators on Hilbert spaces, complemented subspaces, $C_{p}$-spaces. 
Proposition 1.1. Let E be a symmetric sequence space. Then the following are equivalent

(i) $T$ is bounded in $C_{E}$;

(ii) for every $\lambda \neq 1$ the operator $V_{\lambda}=\lambda T+I-T$ is bounded in $C_{E}$;

(iii) $T_{E}$ is isomorphic to $C_{E}$.

Proof. The eigenvalence (i) $\Leftrightarrow$ (ii) follows from the formula

$$
T=\left(I-V_{\lambda}\right) /(1-\lambda) \text {. }
$$

The eigenvalence (i) $\Leftrightarrow$ (iii) is proved in [2].

A triangle is a double sequence of the form $\left\{x_{i, j_{l}}\right\}_{1<i<j<\infty}$. A subtriangle of $\left\{x_{i, j}\right\}_{i<j}$ is a triangle of the form $\left\{x_{i_{k} j_{l}}\right\}_{k<l}$ where $\left\{i_{k}\right\}$ and $\left\{j_{l}\right\}$ are increasing sequences with $i_{k} \leqslant j_{k}$ for every $k$. If we consider a triangle $\left\{x_{i, j}\right\}_{i<j}$ of elements of a Banach space as a basic sequence, we shall always assume that it is a basic sequence in the lexicographic ordering:

$$
x_{1,1}, x_{1,2}, x_{2,2}, x_{1,3}, x_{2,3}, x_{3,3}, \ldots
$$

Definition 1.2. Let $\Delta=\left\{\alpha_{i, j}\right\}_{i<j}$ be a triangle of numbers. We denote by $L(\Delta)$ the set of all numbers $\alpha$ so that for some subtriangle $\left\{\alpha_{i_{k} v_{l}}\right\}_{k<l}$ the following limits exist

$$
\alpha_{k}=\lim _{l \rightarrow \infty} \alpha_{i_{k}, j i}, \quad \alpha=\lim _{k \rightarrow \infty} \alpha_{k} .
$$

Note that if $\Delta$ is bounded, i.e. $\sup _{i, j}\left|\alpha_{i, j}\right|<\infty$, then $L(\Delta) \neq \varnothing$. This can be proved by standard compactness arguments and a diagonal process. Note also that if $\alpha \in L(\Delta)$, then the subtriangle $\left\{\alpha_{i_{k} j_{l}}\right\}_{k<l}$ can be chosen so that the convergence in (1.5) is arbitrarily fast.

2. The main lemma. The following lemma is our main tool.

LEMMA 2.1. Let $E$ be any symmetric sequence space, and let $S: T_{E} \rightarrow C_{E}$ be a nonzero bounded operator. Let $x_{i, j}=S e_{i, j}, \alpha_{i, j}=x_{i, j}(i, j), 1<i<j<\infty$, and let $\alpha \in L\left(\left\{\alpha_{i, j}\right\}_{i<j}\right)$. Then for every $0<\varepsilon<1$ there exist increasing sequences of positive integers $\left\{m_{\nu}\right\}_{\nu=1}^{\infty}$ and $\left\{n_{\nu}\right\}_{\nu=1}^{\infty}$, satisfying $m_{\nu} \leqslant n_{\nu}<m_{\nu+1}$ for every $\nu$, so that if $Q=Q\left(\left\{m_{\nu}\right\},\left\{n_{\nu}\right\}\right)$ is defined by (1.1) and if we define $U=(Q S-\alpha I) T Q$ : $C_{E} \rightarrow C_{E}$, then $\|U\| \leqslant \varepsilon$.

Proof. By passing to a subtriangle if necessary, we may assume that for some numbers $\left\{\alpha_{i}\right\}_{i=1}^{\infty}$, we have

$$
\left|\alpha_{i, j}-\alpha_{i}\right| \leqslant \varepsilon \cdot 8^{-i-j}, \quad\left|\alpha_{i}-\alpha\right| \leqslant \varepsilon \cdot 8^{-i}
$$

Now, for each fixed $i,\left\{e_{i, j}\right\}_{j=i}^{\infty}$ is isometrically equivalent to the unit vector basis of $l_{2}$, so $x_{i, j}=S e_{i, j} \rightarrow 0$ weakly as $j \rightarrow \infty$. By standard perturbation arguments we can assume that for some increasing sequences of positive integers $\left\{\tau_{k}\right\}_{k=1}^{\infty},\left\{i_{k}\right\}_{k=1}^{\infty}$ and $\left\{j_{k}\right\}_{k=1}^{\infty}$ with

$$
\tau_{k}<i_{k} \leqslant j_{k} \leqslant \tau_{k+1}, \quad k=1,2, \ldots,
$$

we have for every $k \leqslant l$,

$$
x_{i_{k} j_{l}}(i, j)=0 \text { if either } \max \{i, j\} \leqslant \tau_{l} \text { or } \max \{i, j\}>\tau_{l+1} .
$$


For every $n, k, l$ with $k<l$ we define

$$
\lambda(n, k, l)=x_{i_{k} j_{l}}\left(i_{n}, j_{l}\right) \text {. }
$$

Note that $\lambda(k, k, l)=\alpha_{i_{k} j_{l}}$, and that in general $|\lambda(n, k, l)|<\left\|x_{i_{k} j_{l}}\right\|<\|S\|$.

We construct now an increasing sequence $\left\{l_{\nu}\right\}_{\nu=1}^{\infty}$ of positive integers and numbers $\lambda(n, k)$ so that for every $n, k<\nu$,

$$
\left|\lambda\left(n, k, l_{\nu}\right)-\lambda(n, k)\right| \leqslant \varepsilon \cdot 8^{-n-k-\nu}
$$

Indeed, every subsequence of $\{\lambda(n, k, l)\}_{l=\max \{n, k\}}^{\infty}$ has a further convergent subsequence. Let $\left\{l_{i}^{(1)}\right\}_{i=1}^{\infty}$ be an increasing sequence, and let $\lambda(1,1)$ be such that $\left|\lambda\left(1,1, l_{i}^{(1)}\right)-\lambda(1,1)\right|<\varepsilon \cdot 8^{-2-i}$ for every $i$. If $\left\{l_{i}^{(m)}\right\}_{i=m}^{\infty}$ and $\{\lambda(n, k)\}_{\max \{n, k\}=m}$ have been defined, let $\{\lambda(n, k)\}_{\max \{n, k\}=m+1}$ be numbers so that for some subsequence $\left\{l_{i}^{(m+1)}\right\}_{i=m+1}^{\infty}$ of $\left\{l_{i}^{(m)}\right\}_{i=m+1}^{\infty}$ we have

$$
\left|\lambda\left(n, k, l_{i}^{(m+1)}\right)-\lambda(n, k)\right|<\varepsilon \cdot 8^{-n-k-i}
$$

for every $i$ and every $n, k$ with $\max \{n, k\}=m+1$. By defining $l_{v}=l_{v}^{(v)}$, we clearly get that (2.5) holds for every $n, k<\nu$.

Note that by (2.1),

$$
\lambda(k, k)=\alpha_{i_{k}} \text { for every } k \text {. }
$$

Let $n_{0}$ and $k$ be given and let $\nu=\max \left\{n_{0}, k\right\}$. Then by (2.5),

$$
\left(\sum_{n=1}^{n_{0}}|\lambda(n, k)|^{2}\right)^{1 / 2}<\left(\sum_{n=1}^{n_{0}}\left|\lambda\left(n, k, l_{v}\right)\right|^{2}\right)^{1 / 2}+\varepsilon<\left\|x_{i_{k} j_{\nu}}\right\|+\varepsilon<\|S\|+\varepsilon .
$$

It follows that for some increasing sequence $\left\{k_{\mu}\right\}_{\mu=1}^{\infty}$ of positive integers and for some subsequence of $\left\{l_{\nu}\right\}_{\nu=1}^{\infty}$ which we continue to denote by $\left\{l_{\nu}\right\}_{\nu=1}^{\infty}$ for convenience, we have $k_{\mu}<l_{\mu}<k_{\mu+1}$ for every $\mu$, and

$$
\left|\lambda\left(k_{\mu}, k_{\nu}\right)\right| \leqslant \varepsilon \cdot 8^{-\mu-\nu} \quad \text { for every } \nu<\mu .
$$

We now claim that

$$
\lambda\left(k_{\mu}, k_{\sigma}\right) \rightarrow 0 \text { as } \sigma \rightarrow \infty \text {, for every } \mu .
$$

Indeed, if (2.10) is false for some $\mu$, then for some $a \neq 0$ and some increasing sequence $\left\{\sigma_{t}\right\}_{t=1}^{\infty}$, we have $\left|\lambda\left(k_{\mu}, k_{\sigma_{t}}\right)-a\right|<2^{-t}$ for every $t$. Let $N$ be such that $\|S\|(N+1)^{1 / 2}<(N+1)|a|-1-\varepsilon$, and choose $\nu$ so that $\nu>\max \left\{k_{\mu}, k_{\sigma_{2 N}}\right\}$. Then, by (2.5),

$$
\begin{aligned}
\|S\|(N+1)^{1 / 2} & \geqslant\left|\sum_{t=N}^{2 N} x_{i_{k_{\sigma_{t}}} j_{l_{\nu}}} \| \geqslant\right| \sum_{t=N}^{2 N} \lambda\left(k_{\mu}, k_{\sigma_{t}}, l_{v}\right) \mid \\
& \geqslant\left|\sum_{t=N}^{2 N} \lambda\left(k_{\mu}, k_{\sigma_{t}}\right)\right|-\sum_{t=N}^{2 N} \varepsilon \cdot 8^{-\mu-\sigma_{t}-\nu} \\
& \geqslant(N+1)|a|-\sum_{t=N}^{2 N} 2^{-t}-\varepsilon>(N+1)|a|-1-\varepsilon .
\end{aligned}
$$

This contradicts the choice of $N$ and thus proves (2.10). 
604

JONATHAN ARAZY

By passing to further subsequences of $\left\{k_{\mu}\right\}_{\mu=1}^{\infty}$ and $\left\{l_{\nu}\right\}_{\nu=1}^{\infty}$ if necessary, we may assume that

$$
\left|\lambda\left(k_{\mu}, k_{\nu}\right)\right|<\varepsilon \cdot 8^{-\mu-\nu}, \quad \mu \neq \nu,
$$

and that

$$
k_{\mu}<l_{\mu}<k_{\mu+1}, \quad \mu=1,2, \ldots
$$

Let for $\mu, \nu=1,2, \ldots$

$$
m_{\mu}=i_{k_{2 \mu-1}}, \quad n_{\nu}=j_{l_{2}}
$$

and let

$$
Q=Q\left(\left\{m_{\nu}\right\},\left\{n_{\nu}\right\}\right)
$$

be the projection defined by (1.1). Note that by (2.2), (2.3), (2.13), (2.14) and (2.15), we have

$$
Q x_{m_{\mu}, n_{3}} \in T_{E}, \quad \mu<\nu .
$$

Define

$$
U=(Q S=\alpha I) T Q
$$

where $T$ is the triangular projection (1.2).

Let $\sum_{i, j=1}^{\infty} t_{i, j} e_{i, j}$ be a normalized element of $C_{E}$, so that $t_{i, j} \neq 0$ only for finitely many pairs $(i, j)$. Then using (in this order) (2.13), (2.14), (2.16), the fact that for fixed $i,\left\{e_{i, j}\right\}_{j=1}^{\infty}$ are isometrically equivalent to the unit vector basis of $l_{2},(2.5),(2.1)$ and (2.12), we get

$$
\begin{aligned}
& \left\|U \sum_{i, j=1}^{\infty} t_{i, j} e_{i, j}\right\|=\left\|\sum_{\mu<\nu} t_{m_{k}, n_{3}}\left(Q x_{m_{k}, n_{3}}-\alpha e_{m_{k}, n_{3}}\right)\right\| \\
& <\left\|\sum_{\mu<\nu} t_{m_{k}, n_{3}}\left(\lambda\left(k_{2 \mu-1}, k_{2 \mu-1}, l_{2 v}\right)-\alpha\right) e_{m_{\mu}, n^{\prime}}\right\| \\
& +\left\|\sum_{\mu<\nu} t_{m_{\nu}, m_{3}} \sum_{\substack{\sigma=1 \\
\sigma \neq \mu}}^{\nu} \lambda\left(k_{2 \sigma-1}, k_{2 \mu-1}, l_{2 v}\right) e_{m_{,}, n_{3}}\right\| \\
& <\sum_{\mu=1}^{\infty}\left(\sum_{\nu=1}^{\infty}\left|t_{m_{\nu}, n_{n}}\right|^{2} \cdot\left|\lambda\left(k_{2 \mu-1}, k_{2 \mu-1}, l_{2 v}\right)-\alpha\right|^{2}\right)^{1 / 2} \\
& +\sum_{\substack{\mu, \sigma=1 \\
\mu \neq \sigma}}^{\infty}\left(\sum_{\nu=\max \{\mu, \sigma\}}^{\infty}\left|t_{m_{\nu}, \mu}\right|^{2} \cdot\left|\lambda\left(k_{2 \sigma-1}, k_{2 \mu-1}, l_{2 v}\right)\right|^{2}\right)^{1 / 2} \\
& <2 \varepsilon \sum_{\mu=1}^{\infty} 8^{-\mu}+2 \varepsilon \sum_{\mu, \sigma=1}^{\infty} 8^{-\mu-\sigma}<2 \varepsilon\left(\frac{1}{7}+\frac{1}{49}\right)<\varepsilon .
\end{aligned}
$$

So, $\|U\|<\varepsilon$ and the Lemma is proved. 
Corollary 2.2. Let $E, S: T_{E} \rightarrow C_{E}$ and $\alpha_{i, j}=\left(S e_{i, j}\right)(i, j), 1<i<j<\infty$, be as in the statement of Lemma 2.1, and assume that $0 \neq \alpha \in L\left(\left\{\alpha_{i, j}\right\}_{i<j}\right)$. Then there exists a subtriangle $\left\{y_{\mu, \nu}\right\}_{\mu<\nu}$ of $\left\{S e_{i, j}\right\}_{i<j}$ which is $2\|S\||\alpha|^{-1}$-equivalent to $\left\{e_{\mu, \nu}\right\}_{\mu<\nu}$ If, moreover, $S\left(T_{E}\right) \subset T_{E}$, then $\left\{y_{\mu, \nu}\right\}_{\mu<\nu}$ can be chosen so that $\left[y_{\mu, \nu}\right]_{\mu<\nu}$ is $2\|S\||\alpha|^{-1}$-complemented in $T_{E}$.

Proof. Let $0<\varepsilon<\min \{|\alpha|,\|S\|\} / 20$. By Lemma 2.1, choose increasing sequences $\left\{m_{\mu}\right\}$ and $\left\{n_{\nu}\right\}$ of positive integers with $m_{\nu}<n_{\nu}<m_{\nu+1}$ for every $\nu$, and so that if we define $Q=Q\left(\left\{m_{\nu}\right\},\left\{n_{\nu}\right\}\right)$ and $U=(Q S-\alpha I) T Q$, then $\|U\|<\varepsilon$. Let $y_{\mu, \nu}=S e_{m_{k}, n_{\nu}}, \mu<\nu$. Then for every $x=\Sigma_{\mu<\nu} t_{\mu, \nu} e_{m_{k}, n_{3}} \in C_{E}$, we have

$$
\begin{aligned}
\left\|\sum_{\mu<\nu} t_{\mu, \nu} y_{\mu, \nu}\right\| & =\|S x\|>\|Q S x\|>|\alpha| \cdot\|x\|-\|Q S x-\alpha x\| \\
& >(|\alpha|-\|U\|)\|x\|>(|\alpha|-\varepsilon)\|x\|>|\alpha| \cdot\|x\| / 2 .
\end{aligned}
$$

Since $\left\|\Sigma_{\mu<\nu} t_{\mu, \nu} y_{\mu, \nu}\right\|=\|S x\|<\|S\| \cdot\|x\|$, we get that $\left\{y_{\mu, \nu}\right\}_{\mu<\nu}$ is $2\|S\||\alpha|^{-1}$ equivalent to $\left\{e_{m_{\mu}, n_{\nu}}\right\}_{\mu<\nu}$. This proves the first assertion since $\left\{e_{m_{\mu}, n_{3}}\right\}_{\mu<\nu}$ is isometrically equivalent to $\left\{e_{\mu, \nu}\right\}_{\mu<\nu}$.

Now assume that $S T_{E} \subset T_{E}$. Let $Y=\left[y_{\mu, \nu}\right]_{\mu<\nu}=S Q T_{E}$, and define

$$
\begin{gathered}
P_{0}=\alpha^{-1}(S Q-U)_{\mid T_{E}}, \\
V=I+\alpha^{-1} U .
\end{gathered}
$$

Clearly, $P_{0}$ is a projection in $T_{E}$ and $\left\|P_{0}\right\|<21\|S\| / 20|\alpha|$. Also, $\|V-I\|<|\alpha|^{-1} \varepsilon$ $<1 / 20$, and so $V$ is an automorphism of $T_{E}$. It follows that

$$
P=V P_{0} V^{-1}
$$

is a projection in $T_{E},\|P\|<\|V\| \cdot\left\|P_{0}\right\| \cdot\left\|V^{-1}\right\|<2\|S\||\alpha|^{-1}$, and since $V P_{0}=$ $\alpha^{-1} S Q_{\mid T_{E}}$, the range of $P$ is exactly $Y$.

3. Proof of Theorem A. We are now ready to prove Theorem $A$ stated in the abstract. We actually prove a somewhat stronger result, namely:

THeORem 3.1. Let $E$ be a symmetric sequence space, let $X$ be either $C_{E}$ or $T_{E}$ and let $P$ be any bounded projection in $X$. Then either $P X$ or $(I-P) X$ contains a subspace which is isomorphic to $X$ and complemented in $X$.

Proof of Theorem 3.1 for $X=T_{E}$. Let us denote for $1<i<j<\infty, a_{i, j}=$ $P e_{i, j}, b_{i, j}=(I-P) e_{i, j}, \alpha_{i, j}=a_{i, j}(i, j)$ and $\beta_{i, j}=b_{i, j}(i, j)$. Since $a_{i, j}+b_{i, j}=e_{i, j}$ for every $i<j$, we have $\alpha_{i, j}+\beta_{i, j}=1$, and thus either $\left|\alpha_{i, j}\right|>1 / 2$ or $\left|\beta_{i, j}\right|>1 / 2$ (or both). By Ramsey's theorem (see [4]) there exists an increasing sequence of positive integers $\left\{i_{k}\right\}_{k=1}^{\infty}$ so that either

$$
\left|\alpha_{i_{k}, i}\right|>1 / 2 \text { for every } k<l \text {, }
$$

or

$$
\left|\beta_{i_{k}, i_{l}}\right|>1 / 2 \text { for every } k<l \text {. }
$$

Without loss of generality we assume that (3.1) holds (since, otherwise (3.2) holds, and the proof is the same provided we replace $P$ by $I-P$ and $a_{i, j}$ by $b_{i, j}$ ). 
Choose any $\alpha \in L\left(\left\{\alpha_{i_{k}, i}\right\}_{k<1}\right)$, and note that (3.1) implies that $|\alpha|>1 / 2$. Using Lemma 2.1 and Corollary 2.2 with $S=P$, we get a subtriangle $\left\{y_{\mu, \nu}\right\}_{\mu<\nu}$ of $\left\{a_{i, i},\right\}_{k<l}$ which is $4\|P\|$-equivalent to $\left\{e_{\mu, v}\right\}_{\mu<\nu}$, and so that $\left[y_{\mu, v}\right]_{\mu<\nu}=Y$ is $4\|P\|$-complemented in $T_{E}$. Since $Y \subset P T_{E}$, this completes the proof in this case.

Proof of Theorem 3.1 for $X=C_{E}$. By the case $X=T_{E}$ treated above, it is enough to consider here only the spaces $C_{E}$ so that $C_{E} \approx T_{E}$. That is, by Proposition 1.1, the spaces $C_{E}$, so that

$$
V_{\lambda}=\lambda T+I-T \text { is bounded in } C_{E} \text { only for } \lambda=1 \text {. }
$$

Let us denote, for every $1<i, j<\infty, a_{i, j}=P e_{i, j}, b_{i, j}=(I-P) e_{i, j}, \alpha_{i, j}=$ $a_{i, j}(i, j)$ and $\beta_{i, j}=b_{i, j}(i, j)$. Using Ramsey's theorem once again, we get an increasing sequence $\left\{i_{k}\right\}_{k=1}^{\infty}$ so that either

$$
\left|\alpha_{i_{k}, i}\right|>1 / 2 \text { for every } l<k,
$$

or

$$
\left|\beta_{i_{k}, i}\right| \geqslant 1 / 2 \text { for every } l<k .
$$

Again, we assume without loss of generality that (3.4) holds. By passing to a subsequence of $\left\{i_{k}\right\}_{k=1}^{\infty}$ if necessary we may assume also that for some $\alpha$ with $1 / 2<|\alpha|<\|P\|$ we have $L\left(\left\{\alpha_{i_{k}, i}\right\}_{l<k}\right)=\{\alpha\}$. Choose some $\gamma \in L\left(\left\{\alpha_{i_{k}, i}\right\}_{k<l}\right)$ and let

$$
0<\varepsilon<\|P\| / 20(\|P\|+1) .
$$

Applying Lemma 2.1 to $S_{1}=P_{\mid T_{E}}$ and $T_{E}$ we construct subsequences $\left\{n_{\nu}^{(1)}\right\}_{\nu=1}^{\infty}$ and $\left\{m_{\nu}^{(1)}\right\}_{\nu=1}^{\infty}$ of $\left\{i_{k}\right\}_{k=1}^{\infty}$ so that $m_{\nu}^{(1)}<n_{\nu}^{(1)}<m_{\nu+1}^{(1)}$ for every $\nu$ and so that if $Q_{1}=$ $Q\left(\left\{m_{\nu}^{(1)}\right\},\left\{n_{\nu}^{(1)}\right\}\right)$, then

$$
\left\|\left(Q_{1} S_{1}-\gamma I\right) T Q_{1}\right\|<\varepsilon .
$$

Clearly, $L\left(\left\{\alpha_{m_{m}^{(1)}, m^{(1)}}\right\}_{\nu<\mu}\right)=\{\alpha\}$. Now $T_{E}$ is isometric in a natural way to $\tilde{T}_{E}=$ $\left[e_{i, j}\right]_{j<i}$ so by applying Lemma 2.1 to $S=P_{\mid \tilde{T}_{E}}$ and to $\tilde{T}_{E}$ and $I-T$ instead of $T_{E}$ and $T$ respectively, we get subsequences $\left\{n_{v}\right\}_{v-1}^{\infty}$ and $\left\{m_{v}\right\}_{v-1}^{\infty}$ of $\left\{n_{\nu}^{(1)}\right\}_{v-1}^{\infty}$ and $\left\{m_{\nu}^{(1)}\right\}_{\nu=1}^{\infty}$ respectively, with $m_{\nu}<n_{\nu}<m_{\nu+1}$ for every $\nu$, so that if $Q=$ $Q\left(\left\{m_{v}\right\},\left\{n_{v}\right\}\right)$, then

$$
\|(Q S-\alpha I)(I-T) Q\|<\varepsilon .
$$

Since the family $\left\{Q, Q_{1}, T\right\}$ is commutative, it is clear that (3.7) holds also with $Q$ instead of $Q_{1}$. Let

$$
W=V_{(\gamma / \alpha)} \cdot Q=(\gamma T / \alpha+I-T) Q .
$$

Then for every $x \in C_{E}$ with only finitely many nonzero coordinates,

$$
\begin{aligned}
\left\|\left(\alpha^{-1} Q P Q-W\right) x\right\| & <\left\|\left(Q S_{1}-\gamma I\right) T Q x\right\| /|\alpha|+\|(Q S-\alpha I)(I-T) Q x\| /|\alpha| \\
& <2 \varepsilon\|x\| /|\alpha| .
\end{aligned}
$$

In particular this implies that $W$ is bounded in $C_{E}$. Let $J$ be the isometry of $C_{E}$ onto $Q C_{E}$ defined by $J e_{\mu, \nu}=e_{m_{,}, \eta_{3}}$, then $J^{-1} W J=V_{(\gamma / \alpha)}$. By the assumption (3.3) we get that $\alpha=\gamma$ and thus $W=Q$. 
Let $U=\alpha^{-1} P J$. Then for every $x \in C_{E}$,

$\|U x\|>\|Q U x\|>\|J x\|-\left\|\alpha^{-1} Q P Q J x-Q J x\right\|>\left(1-2 \varepsilon|\alpha|^{-1}\right)\|x\|>\frac{4}{5}\|x\|$.

It follows that $U$ is an isomorphism (with constant $<20\|P\| / 9$ ) from $C_{E}$ onto some subspace $Z$ of $P C_{E}$. Let

$$
\begin{gathered}
R_{0}=\alpha^{-1} P Q+Q-\alpha^{-1} Q P Q \\
V=I+\alpha^{-1} Q P Q-Q
\end{gathered}
$$

Then $R_{0}$ is a bounded projection in $C_{E}$, and by (3.10), $\|V-I\|<1 / 5$. So, $V$ is an automorphism of $C_{E}$ and $R=V R_{0} V^{-1}$ is a projection in $C_{E}$ with $\|R\|<4\|P\|$. Since $V R_{0}=\alpha^{-1} P Q$, the range of $R$ is $Z$. This completes the proof of Theorem 3.1.

4. Applications. Recall that a Banach space $X$ is primary if for every bounded projection $P$ on $X$, either $P X \approx X$ or $(I-P) X \approx X$. For $1<p<\infty$, let $C_{p}=C_{\zeta}$, and let $C_{\infty}=C_{c_{0}}$. It is known that for $1<p<\infty, C_{p}$ is primary (see [1]). We strengthen this somewhat as follows.

Corollary 4.1. Let $C_{E}$ be a unitary matrix space so that for some $1<p<\infty$,

$$
C_{E} \approx\left(C_{E} \oplus C_{E} \oplus \cdots \oplus C_{E} \oplus \cdots\right)_{\zeta}
$$

(where if $p=\infty$, the direct sum is taken in the sense of $c_{0}$ ). Then $C_{E}$ is primary. In particular, the spaces $C_{p}, 1 \leqslant p \leqslant \infty$, are primary.

The corollary follows easily from Theorem 3.1, the decomposition method (see [6, p. 54]) and the fact (see [1]) that for every $1<p<\infty$,

$$
C_{p} \approx\left(C_{p} \oplus C_{p} \oplus \cdots \oplus C_{p} \oplus \cdots\right)_{l_{p}}
$$

These arguments show that Corollary 4.1 holds also for $T_{E}$ instead of $C_{E}$.

Problem 4.2. Is every unitary matrix space a primary Banach space?

Let us pass to a local (i.e. finite dimensional) version of Theorem 3.1. If $E$ is a symmetric sequence space, then $C_{E}^{n}$ denotes the space of all $n \times n$ matrices with the norm induced from $C_{E}$. Let $\left\{A_{n}\right\}_{n=1}^{\infty}$ be pairwise disjoint subsets of the positive integers so that $A_{n}$ has exactly $n$ elements, and let

$$
S_{E}=\left\{x \in C_{E} ; x(i, j)=0 \text { if }(i, j) \notin \bigcup_{n=1}^{\infty} A_{n} \times A_{n}\right\} .
$$

$S_{E}$ can be considered in the obvious way as the direct sum $S_{E}=\left(\sum_{n=1}^{\infty} \oplus C_{E}^{n}\right)_{E}$. The importance of $S_{E}$ stems from the easily proved fact that $C_{E}$ is finitely represented in $S_{E}$.

Using the same ideas as in the proofs of Lemma 2.1 and Theorem 3.1, we are able to prove the following results. 
COROLlary 4.2. Let $k$ be a positive integer and let $1<M<\infty$. Then there exists a positive integer $n=n(k, M)$ so that if $E$ is any symmetric sequence space and if $P$ is any projection in $C_{E}^{n}$ with $\|P\|<M$, then either $P C_{E}^{n}$ or $(I-P) C_{E}^{n}$ contains a subspace which is $4(M+1)$-isomorphic to $C_{E}^{k}$ and $4(M+1)$-complemented in $C_{E}^{n}$.

COROLlaRY 4.3. Let $E$ be any symmetric sequence space and let $P$ be a bounded projection in $S_{E}$. Then either $P S_{E}$ or $(I-P) S_{E}$ contains a subspace which is isomorphic to $S_{E}$ and complemented in $S_{E}$.

Corollary 4.4. For $1<p<\infty$, the space $S_{P}=\left(\sum_{n=1}^{\infty} \bigoplus C_{p}^{n}\right)_{\zeta}$ is primary.

Here, as before, if $p=\infty$ the direct sum is taken in the sense of $c_{0}$.

In proving Corollary 4.2 we use Ramsey's theorem for finite sets many times. The estimate on the growth of $n$ as a function of $k$ we obtain is therefore very bad. In proving Corollary 4.3 , we also use the fact (easily proved by the decomposition method) that if $\left\{n_{k}\right\}_{k=1}^{\infty}$ is any sequence of positive integers so that $\sup _{k} n_{k}=\infty$, then $S_{E} \approx\left(\sum_{k=1}^{\infty} \oplus C_{E}^{n_{k}}\right)_{E}$.

Problem 4.5. Let $E$ be a symmetric sequence space. Is $S_{E}$ a primary Banach space?

\section{REFERENCES}

1. J. Arazy, On large subspaces of Schatten p-classes, Compositio Math. (to appear).

2. Some remarks on interpolation theorems and the boundness of the triangular projection in unitary matrix spaces, Integral Equations Operator Theory 1 (1978), 453-495.

3. I. C. Gohberg and M. G. Krein, Introduction to the theory of linear non self adjoint operators, Transl. Math. Mono., vol. 18, Amer. Math. Soc., Providence, R. I., 1969; reprinted 1978.

4. M. Hall, Jr., Combinational theory, Blaisdell, Waltham, Mass., 1967.

5. S. Kwapien and A. Pel'czynski, The main triangle projection in matrix spaces and its applications, Studia Math. 34 (1970), 43-68.

6. J. Lindenstrauss and L. Tzafriri, Classical Banach spaces 1, sequences spaces, Springer-Verlag, Berlin and New York, 1977.

Department of Pure Mathematics, The Weizmann Institute of Science, Rehovot, Israbi

Current address: Department of Mathematics, University of Illinois at Urbana-Champaign, Urbana, Illinois 61801 